\title{
Different Estimates of Cardiac Power: Relationship to Altered Loading Conditions
}

\author{
MS Firstenberg, G Armstrong, NL Greenberg, MJ Garcia, JD Thomas
}

The Cleveland Clinic Foundation, Cleveland, United States

\begin{abstract}
Peak instantaneous power is a prognostic index of $L V$ function but is difficult to measure. It is relatively load independent when corrected by preload $\left(E D V^{2}\right)$. Easier to acquire power estimates have been proposed - including mean and simplified power. These require only echo Doppler flow and cuff pressures, but their relationship to invasively derived results has not been studied.

Using a well-validated numerical model of the cardiovascular system, different estimates of power were compared for varying preload, afterload, and contractility. All power results were divided by $E D V^{2}$.

Estimates of power correlate with peak power over a wide range of physiology. Corrected power estimates were independent of preload and afterload, but predictably increased with contractility.

Different estimates of preload corrected power can be derived from non-invasively obtained variables and correlate with corrected peak instantaneous power.
\end{abstract}

\section{Introduction}

A preload independent index of left ventricular (LV) contractility remains the Holy Grail of the non-invasive assessment of left ventricular function [1]. While many different indices as derived from various imaging modalities have been proposed, most rely on complex analysis of echocardiographic images or a combination of non-invasive and invasive hemodynamic data. Ventricular power has been shown to be a valuable index of LV contractility and is derived as the instantaneous product of pressure and flow. Accurate measurements require invasive measurements of aortic root (or direct $\mathrm{LV}$ ) pressures combined with temporal reconstructions of aortic flow from LV outflow tract Doppler waveforms. Obviously, this is technically demanding, time consuming, and impractical for routine clinical use. Various other means of estimating cardiac power (peak and/or mean) have been proposed, but little work has been done validating the methods against peak instantaneous power (PIP) [2].

Furthermore, it has been shown that when corrected by end-diastolic volume (EDV), and even more-so by EDV squared $\left(\mathrm{EDV}^{2}\right)$, PIP can be a relatively preloadindependent index of LV contractility - hence enhancing it's application to the assessment of cardiac function independent of loading conditions [2,3]. The utility of non-invasive estimates of cardiac power would therefore be enhanced if they too were shown to be preloadindependent when corrected by either EDV or EDV ${ }^{2}$.

\section{Methods}

\subsection{Numerical modeling}

A numerical model of the cardiovascular system was developed based upon fluid dynamics concepts and previous research describing the mathematical relationships of cardiac chamber (atrial and ventricular) systolic and diastolic pressure-volume curves. Our model, written in the LabVIEW (National Instruments, Austin, TX, USA) programming environment, is a lumped parameter, closed-loop model that consist of 24 first order differential equations. These equations are solved iteratively using the fourth-order Runge-Kutta method and results in instantaneous $(5 \mathrm{~ms}$ intervals $)$ pressures (Equation 1), volumes (Equation 2), and flows (Equation 3) through the heart and cardiovascular system.

Equation 1: $\quad \frac{d P_{i}}{d t}=\frac{\left(Q_{i-1 / 2}-Q_{i+1 / 2}\right)}{C_{i}}$

Equation 2: $\quad \frac{d V}{d t}=Q_{\text {in }}-Q_{\text {out }}$

Equation 3:

$$
\frac{d Q_{j}}{d t}=\frac{P_{j-1 / 2}-P_{j+1 / 2}-r_{j}\left(Q_{j}\right)}{m_{j}}
$$

Equation Legend:

$\mathrm{C}=$ Compliance

$\mathrm{P}=$ Pressure

$\mathrm{Q}=$ Flow

$\mathrm{V}=$ Volume

$\mathrm{i}=$ Chamber node

$\mathrm{j}=$ Flow node

$\mathrm{m}=$ Inertial term

$\mathrm{r}=$ Resistance term 
The pulmonary and systemic venous and arterial systems were modeled with a linear pressure/volume relationship and compliance. A linear pressure/volume relationship and constant elastance was used for the atria. For the ventricles, a linear P/V relationship was used for systole, whereas diastole was modeled with a rising mono-exponential function above and a negative exponential equation below an equilibrium volume. Experimentally obtained and clinically verified values for left atrial and ventricular systolic and diastolic parameters were used as constants [4].

To explore the effects of changes in preload on the different power equations/relationships, total systemic volume was altered $(4000-6000 \mathrm{ml}$ in $250 \mathrm{ml}$ increments). Similarly, to explore the relationships under varying afterload conditions, aortic compliance was independently varied from 1.0 to $2.5 \mathrm{ml} / \mathrm{mmHg}$. Finally, the independent effects of changes in ventricular function were also studied with a range in ventricular elastance from 2 to $8 \mathrm{mmHg} / \mathrm{ml}$. For each physiologic parameter modeled, waveforms and volumes were analyzed similar to the analysis performed on the clinical waveforms and volumes. Overall, 30 different conditions were modeled.

\subsection{Power calculations}

From the results of numerical modeling, power estimates were derived from the following 5 equations representing both invasive and non-invasive techniques:

$$
\begin{aligned}
& \text { Peak Instantaneous Power }=\mathrm{AoP} \bullet \mathrm{AoF} \\
& \begin{array}{l}
\text { Simplified Power I }=\mathrm{MAP} \bullet \mathrm{AoF} \\
\text { Simplified Power II }=\mathrm{SBP} \bullet \mathrm{AoF}
\end{array} \\
& \begin{array}{l}
\text { Mean Power I }=\mathrm{MAP} \bullet \mathrm{SV} \\
\text { Mean Power II }=\mathrm{SBP} \bullet \mathrm{SV}
\end{array}
\end{aligned}
$$

where AoP and AoF are peak aortic pressure and flow, MAP is mean arterial pressure, SBP is systolic blood pressure, and SV is stroke volume.

\subsection{Statistical analysis}

Each of the 5 power estimates were corrected by EDV and $E^{2} V^{2}$. To allow for direct comparison of the different techniques of determining power, all results were normalized to baseline (i.e. results obtained prior to changes in preload, afterload, or contractility). The slopes, as determined using regression analysis, of each method under each set of changes in physiologic conditions were determined. The slopes were determined for the raw (uncorrected) and corrected (EDV and EDV ${ }^{2}$ ) results.

\section{Results}

\subsection{Perturbations in preload}

The effects of perturbations in preload are demonstrated below:
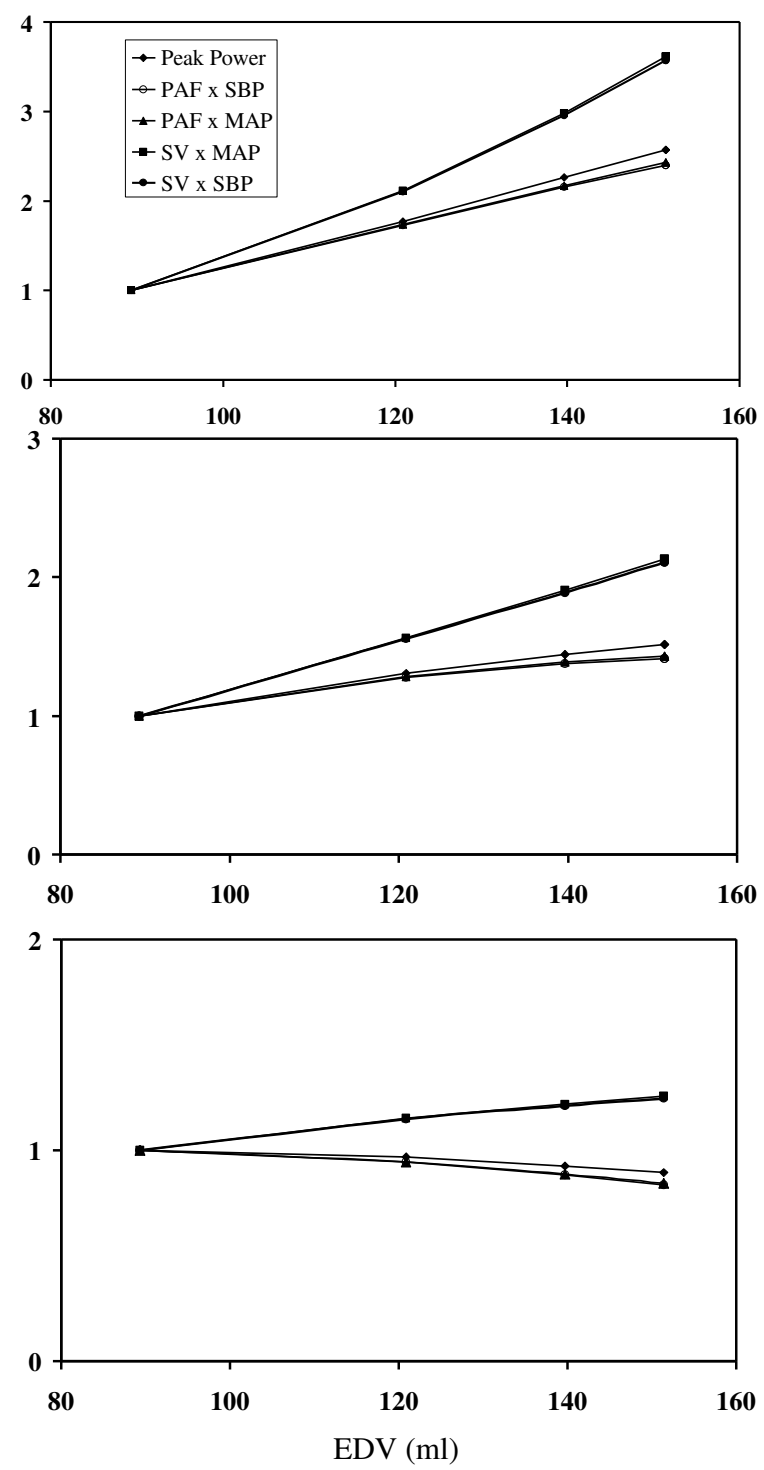

Figure 1a-c: Power estimates as a function of preload (EDV) with (a) no correction, (b) correction by EDV and (c) correction by $\mathrm{EDV}^{2}$. These results are consistent with known Frank-Starling mechanisms. A relative index of cardiac power can be derived and is minimally influenced by changes in preload. 


\subsection{Perturbations in afterload}

The effects of perturbations in afterload are shown below:
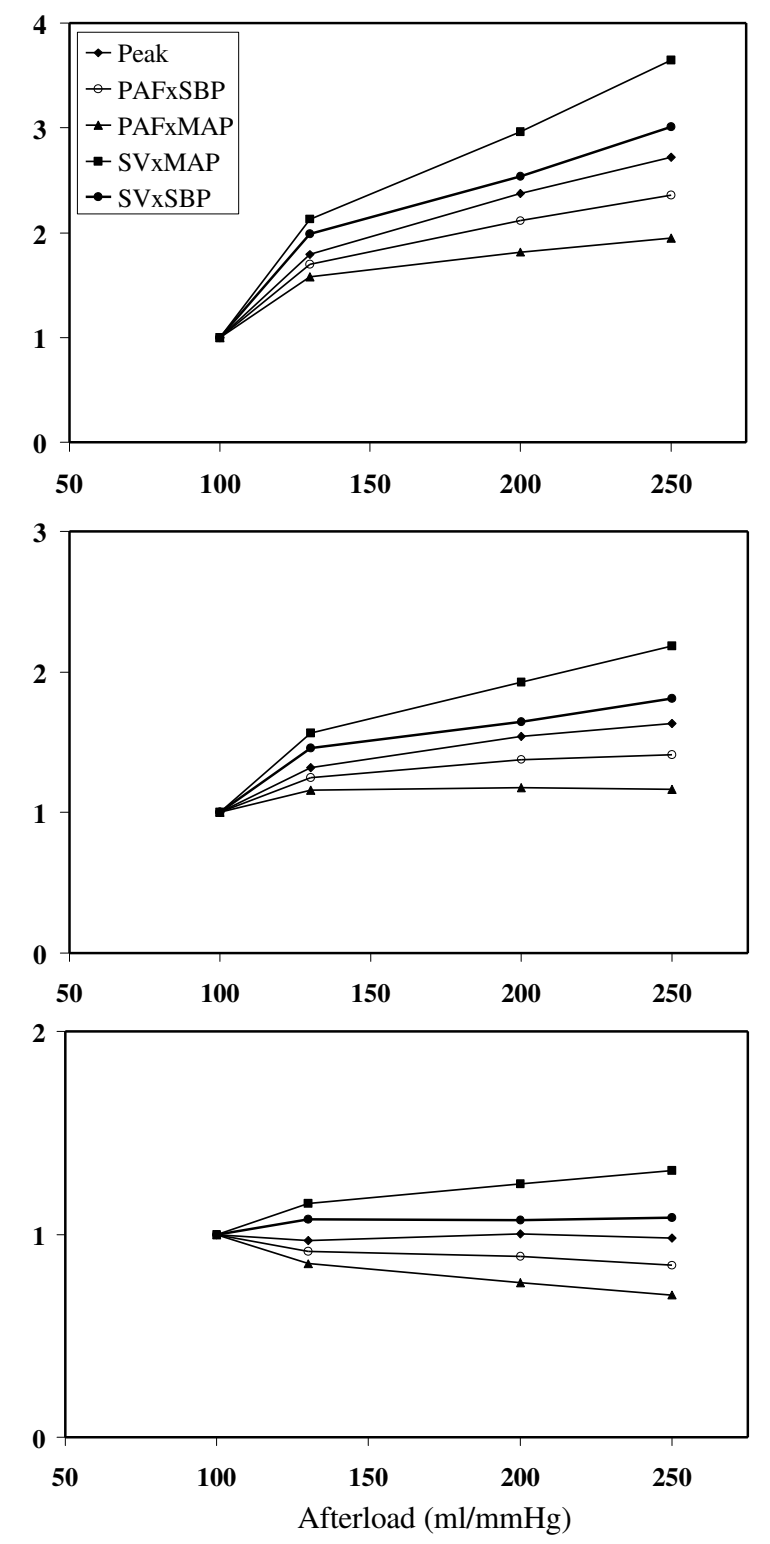

Figure 2a-c: Similarly, top graph (a) shows the relative increases in cardiac power determined by each method with increases in afterload. Again, increases in afterload result in increases in cardiac power. However, the middle graph (b) illustrates the effects of correcting by EDV with the bottom graph showing correction by $\mathrm{EDV}^{2}$, demonstrating a relatively preload-independent index.

\subsection{Perturbations in contractility}

Finally, the effects of changes in contractility are demonstrated below:
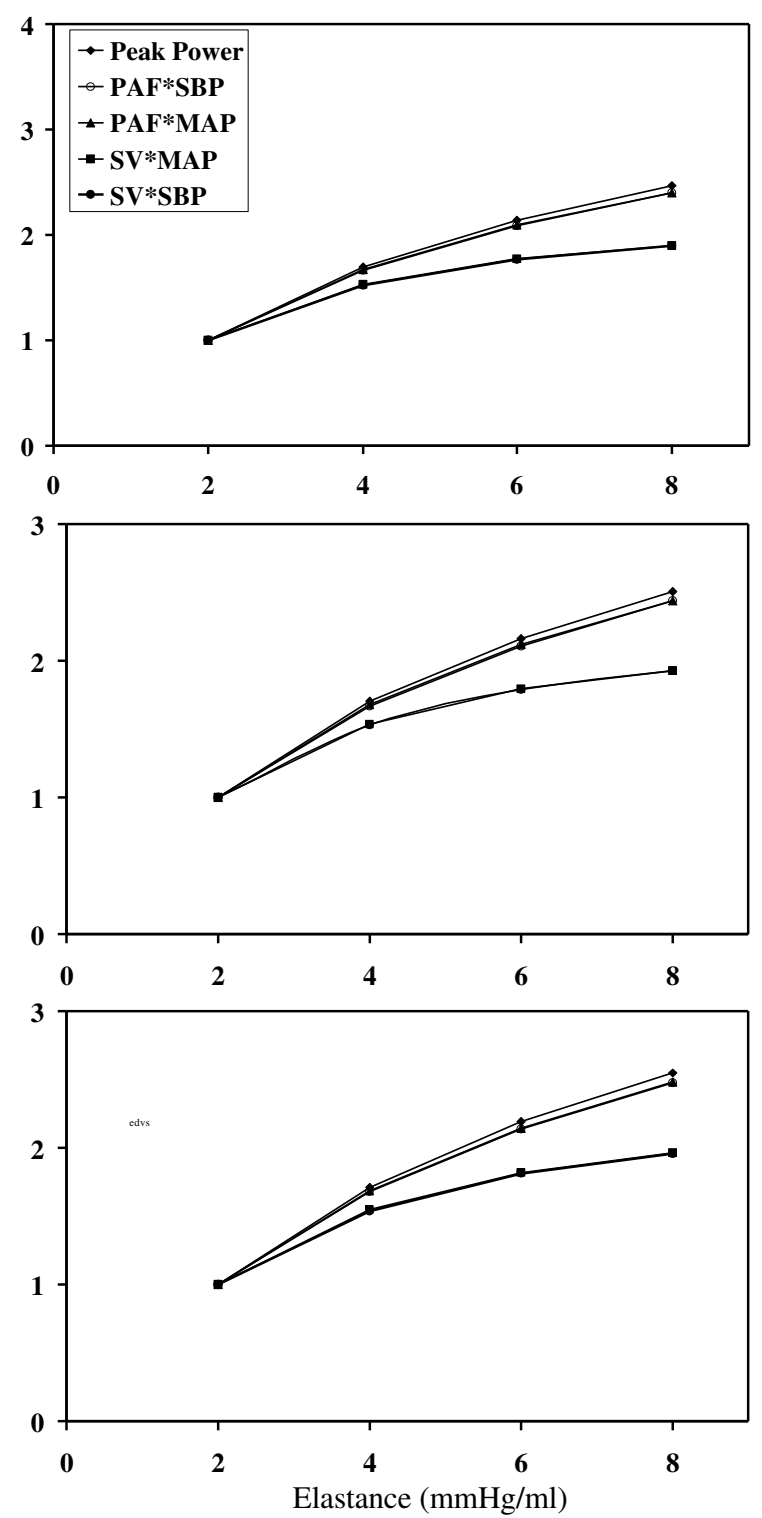

Figure 3a-c: Similarly, top graph (a) shows the relative increases in cardiac power, as determined by each of 5 methods with increases in contractility (LV elastance). Predictably, increases in contractility result in increases in cardiac power mechanisms. Furthermore, these power vs. contractility relationships are preserved when correcting by preload (figure $b$ and $c$ ).

\subsection{Quantitative assessment}

To correlate different methods of estimating power with PIP, the maximal uncorrected and corrected normalized value was determined. Results closest to 1.00 represent a greater degree of independence from the dependent variable. See text for abbreviation descriptions. 
Table 1: Changes in Preload

\begin{tabular}{|l|c|c|c|}
\hline \multirow{2}{*}{ Method } & \multicolumn{3}{|c|}{ Correction Factor } \\
& 1 & $1 / \mathrm{EDV}$ & $1 / \mathrm{EDV}^{2}$ \\
\hline Power (PIP) & 2.57 & 1.52 & 0.90 \\
\hline PAF x SBP & 2.40 & 1.42 & $\mathbf{0 . 8 4}$ \\
\hline PAF x MAP & 2.43 & 1.43 & $\mathbf{0 . 8 4}$ \\
\hline SV x MAP & 3.62 & 2.13 & 1.26 \\
\hline SV x SBP & 3.58 & 2.11 & 1.25 \\
\hline
\end{tabular}

Changes in preload resulted in a 2.57 -fold increase in PIP. When preload corrected, the normalized results closest approximating PIP was PAF x SBP and PAF x MAP.

Table 2: Changes in Afterload

\begin{tabular}{|l|c|c|c|}
\hline Method & Power & $1 / E D V$ & $1 / \mathrm{EDV}^{2}$ \\
\hline Power (PIP) & 2.72 & 1.63 & 0.98 \\
\hline PAF x SBP & 2.36 & 1.41 & $\mathbf{0 . 8 5}$ \\
\hline PAF x MAP & 1.95 & 1.17 & 0.70 \\
\hline SV x MAP & 3.64 & 2.19 & 1.31 \\
\hline SV x SBP & 3.01 & 1.81 & $\mathbf{1 . 0 8}$ \\
\hline
\end{tabular}

Changes in afterload resulted in a 2.72 -fold increase in power. When preload corrected, the normalized result best approximating PIP was PAF x SBP and SV x SBP.

Table 3: Changes in Contractility

\begin{tabular}{|l|c|c|c|}
\hline Method & Power & $1 / \mathrm{EDV}$ & $1 / \mathrm{EDV}^{2}$ \\
\hline Power (PIP) & 2.47 & 2.51 & 2.55 \\
\hline PAF x SBP & 2.40 & 2.44 & $\mathbf{2 . 4 8}$ \\
\hline PAF x MAP & 2.40 & 2.44 & $\mathbf{2 . 4 8}$ \\
\hline SV x MAP & 1.90 & 1.93 & 1.96 \\
\hline SV x SBP & 1.90 & 1.93 & 1.96 \\
\hline
\end{tabular}

Changes in contractility resulted in a 2.47 -fold increase in power. As expected for a load independent index of contractility, results were similar to uncorrected results.

\section{Discussion}

PIP is the peak instantaneous product of LV outflow and pressure during systole. "Preload adjusted" PIP is relatively independent of loading conditions and is predictive of outcome [5]. However, measurement of PIP requires temporal alignment of the LV pressure and outflow Doppler waveforms - both time-consuming and technically demanding.

Other estimates of PIP have been proposed, but each has limitations to clinical use. Maximal power, derived from the peak LV pressure and peak flow, is a potentially useful measure of LV systolic function but has not yet found widespread clinical application in part due to the need for invasive measurement. Mean power, relies on echocardiographic measurement of the aortic time velocity integral instead of peak aortic flow velocity, and is unreliable during exercise echocardiography [6].

Despite these limitations, the invasive and noninvasive assessment of cardiac power can be a useful clinical tool. We have demonstrated, using a well validated numerical model, that different techniques for estimating PIP respond to changes in preload, afterload, and contractility in similar fashion. Furthermore, we have shown that these relationships are preserved when correcting for $\mathrm{EDV}$ and $\mathrm{EDV}^{2}$ thereby numerically validating their potential for broader application.

Although our results are limited by a lack of clinical validation, the application of an established numerical model demonstrates that the results are valid on a theoretical and mathematical basis. Nevertheless, clinical validation is still required.

\section{Conclusions}

Through the application of numerical modeling, different methods for estimating PIP based upon invasively and non-invasively derived parameters correlate with PIP. When preload corrected, similar to true PIP, they are also relatively load-independence.

Simplified techniques of estimating PIP can be used as useful surrogates of overall cardiac contractile function.

\section{References}

[1] Shin H, Schiller NB. Progress in developing a noninvasive load-independent marker of ventricular contractility. $J$ Am Coll Card 1998;31:869-70.

[2] Armstrong GP, Carlier SG, Fukamachi K, Thomas JD, Marwick TH. Estimation of cardiac reserve by peak power: validation and initial application of a simplified index. Heart 1999 Sep;82(3):357-64

[3] Sharir T, Feldman MD, Haber H, Feldman AM, Marmor A, Becker LC, et al. Ventricular systolic assessment in patients with dilated cardiomyopathy by preload-adjusted maximal power. Validation and noninvasive application. Circulation 1994;89:2045-53.

[4] Thomas JD, Zhou J, Greenberg N, Bibawy G, McCarthy PM, Vandervoort PM. Physical and physiological determinants of pulmonary venous flow: numerical analysis. Am J Physiol 1997;272:H2453-H2465.

[5] Marmor A, Schneeweiss A. Prognostic value of noninvasively obtained LV contractile reserve in patients with severe heart failure. J Am Coll Card 1997;29:422-8.

[6] Tan LB, Littler WA. Measurement of cardiac reserve in cardiogenic shock: implications for prognosis and management. Br Heart J 1990;64:121-8.

Address for correspondence.

Michael S. Firstenberg, M.D.

Department of Cardiology, Desk F-15

9500 Euclid Avenue

Cleveland, Ohio 44195 USA

E-mail: firstem@ccf.org 